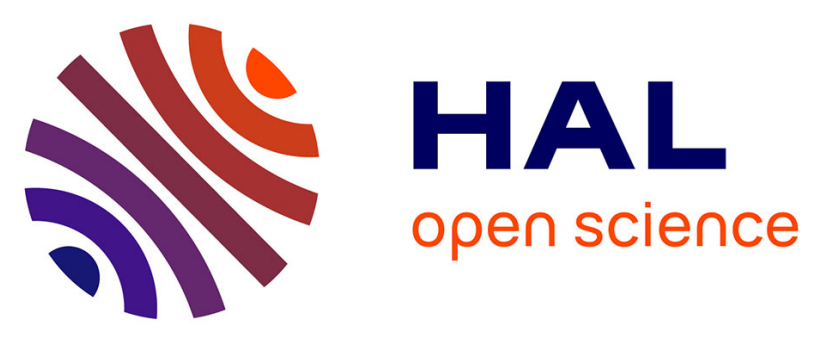

\title{
Evaluation of an 'all-in-one' seven-day whole-genome sequencing solution in the investigation of a Staphylococcus aureus outbreak in a neonatal intensive care unit
}

\author{
C. Rouard, N. Bourgeois-Nicolaos, L. Rahajamanana, O. Romain, L. Pouga, \\ V. Derouin, D. de Luca, F. Doucet-Populaire
}

\section{To cite this version:}

C. Rouard, N. Bourgeois-Nicolaos, L. Rahajamanana, O. Romain, L. Pouga, et al.. Evaluation of an 'all-in-one' seven-day whole-genome sequencing solution in the investigation of a Staphylococcus aureus outbreak in a neonatal intensive care unit. Journal of Hospital Infection, 2019, 102, pp.297 303. 10.1016/j.jhin.2019.01.029 . hal-03486705

\section{HAL Id: hal-03486705 https://hal.science/hal-03486705}

Submitted on 20 Dec 2021

HAL is a multi-disciplinary open access archive for the deposit and dissemination of scientific research documents, whether they are published or not. The documents may come from teaching and research institutions in France or abroad, or from public or private research centers.
L'archive ouverte pluridisciplinaire HAL, est destinée au dépôt et à la diffusion de documents scientifiques de niveau recherche, publiés ou non, émanant des établissements d'enseignement et de recherche français ou étrangers, des laboratoires publics ou privés.

\section{(ㅇ)(1) $\$$}

Distributed under a Creative Commons Attribution - NonCommerciall 4.0 International 


\section{Evaluation of an "all-in-one" seven-day WGS solution in the investigation of a Staphylococcus aureus outbreak in a neonatal Intensive Care Unit}

Caroline Rouard ${ }^{1,2}$, Nadège Bourgeois-Nicolaos ${ }^{1,2,3}$, Lalaina Rahajamanana ${ }^{1 \text {, }}$, Olivier Romain ${ }^{4}$, Lydia Pouga ${ }^{1}$, Véronique Derouin ${ }^{1}$, Daniele De Luca ${ }^{4,5}$, Florence DoucetPopulaire ${ }^{1,2,3}$

\section{${ }^{1}$ AP-HP, Hôpitaux Universitaires Paris Sud, Hôpital Antoine Béclère, Service de} Bactériologie-Hygiène, Clamart, France

${ }^{2}$ EA4043, Bactéries pathogènes et santé, Faculté de Pharmacie, Université Paris Sud, Chatenay-Malabry, France

${ }^{3}$ Institute of Integrative Biology of the Cell, CNRS, CEA, Univ. Paris Sud, Paris Saclay University, Gif sur Yvette, France

${ }^{4}$ AP-HP, Hôpitaux Universitaires Paris Sud, Hôpital Antoine Béclère, Division of Pediatrics and Neonatal Intensive Care, Clamart, France

${ }^{5}$ INSERM-U999 LabEx - LERMIT, Clamart, France.

Corresponding author: Florence Doucet-Populaire, Hôpital Antoine Béclère APHP, Service de Bactériologie-Hygiène, 157 rue de la Porte de Trivaux, 92141 Clamart Cedex, France ; +33145374298; florence.doucet-populaire@aphp.fr

\# Present address: CHU Mère Enfant Tsaralalàna, Service Laboratoire, Antananarivo 101, Madagascar

Running title: S. aureus outbreak investigation in NICU 
Keywords: Staphylococcus aureus, sepsis, outbreak, whole genome sequencing, bioMérieux EpiSeq, neonatal intensive care unit 


\section{Summary}

\section{Background}

Meticillin-susceptible and resistant Staphylococcus aureus (MSSA and MRSA) are responsible for outbreaks in intensive care units. MSSA infections have the same morbidity and mortality rate as MRSA infections but are less often studied. Whole genome sequencing (WGS) is increasingly used for outbreak monitoring but still requires specific installation and trained personnel to obtain and analyse the data.

Aim

To evaluate the workflow and the benefits of the bioMerieux Episeq® (bioMerieux, France) solution in exploring an increased incidence of $S$. aureus bloodstream infections in a neonatal intensive care unit (NICU).

\section{Methods}

Four bacteraemia and 27 colonizing $S$. aureus isolates from January to July 2016 were submitted to the "all in one solution" Episeq ${ }^{\circledR}$ (whole genome sequencing, quality data assessment, MLST, spa type, virulome, resistome and phylogenetic tree construction). More in-depth analyses were performed (wgMLST and wgSNP analysis) with BioNumerics software (Applied Maths, Belgium).

Findings

Nine different STs and 13 different spa types were found among the 31 isolates studied. Among those isolates, $\mathrm{n}=11$ (7 patients) were ST146 spa type t002, $\mathrm{n}=5$ (4 patients) ST30 
and $n=4$ (4 patients) ST398. The eleven ST146 isolates had a maximum of 7 pairwise SNP differences.

Conclusion

The use of the Episeq solution allowed us to quickly demonstrate the polyclonal profile of the MSSA population in neonates and thus rule out the suspicion of a global outbreak. However, wgSNP analysis showed the transmission and persistence of one ST over 6 months in the NICU and enabled the infection control team to adapt its response.

\section{Abbreviations}

ARD antimicrobial resistance determinants

LOS: late onset sepsis

MLST: multilocus sequence typing

MSSA: meticillin-susceptible Staphylococcus aureus

MRSA meticillin-resistant Staphylococcus aureus

NICU: neonatal intensive care unit

SNPs: single nucleotide polymorphisms

ST: sequence type

VISA vancomycin intermediate $S$. aureus

VLBW very low birth weight

WGS: whole genome sequencing

wgMLST: whole genome MLST

wgSNP: whole genome SNP 


\section{INTRODUCTION}

Staphylococcus aureus is the second most common cause of late onset sepsis (LOS) after coagulase negative staphylococci within the neonatal intensive care unit (NICU) population [1-4]. Although the incidence of LOS has decreased, 34\% of very low birth weight children (VLBW) will experience at least one episode [1]. LOS has been associated with a mortality rate of $12 \%$ while increased neurodevelopmental impairment is observed in the surviving infants [1]. S. aureus colonization may be acquired by neonates from healthcare workers, parents and visitors, or inanimate objects in the environment [5]. Meticillinsusceptible $S$. aureus (MSSA) infections have an equivalent morbidity and mortality in preterm infants and are more frequent than meticillin-resistant $S$. aureus (MRSA) infections $[6,7]$. However, due to their antibiotic resistance, MRSA outbreaks are more frequently reported in NICUs. Recently, studies have used whole genome sequencing (WGS) for MRSA outbreak investigation in adults and also in neonates [8,9]. Indeed, the discriminatory power of WGS in analysing isolate relatedness as well as the possibility of predicting resistance and virulence phenotypes is extremely advantageous. As a result, routine use of this technology is being implemented worldwide. However, acquiring WGS data is not easy for every hospital laboratory and the standardization of WGS remains challenging. In response to this, the new bioMerieux Episeq ${ }^{\circledR}$ solution (bioMerieux, Marcy l'Etoile, France), dedicated to the epidemiological monitoring and control of nosocomial infections by WGS, has been introduced. This solution combines a service for WGS (subculture, DNA extraction, library preparation, sequencing run) with secure cloud-based software for data analysis (reads assembly, MLST and spa typing, resistome and virulome characterization, core genome SNPs-based phylogeny). Analysis involves several knowledge bases, including a proprietary and curated collection of well-characterized epidemic $S$. aureus isolates associating genomic and epidemiological data. All of these data are provided directly to the client via intuitive 
software. Moreover, WGS data could be used for whole genome MLST (wgMLST) analysis which could also represent an answer to the problem of standardization. The United States Center for Disease Control (CDC) has recently validated the use of wgMLST and the E. coli genotyping plug-in tool employing the commercial software platform BioNumerics (Applied Maths, Sint-Martens-Latem, Belgium). This tool has the advantage of combining quality assessment, data analysis and databasing of metadata in a single platform [10]. A S. aureus wgMLST scheme has also been developed for BioNumerics and could be used in outbreak investigation as recently evidenced [8].

The goal of our study was to evaluate the relevance and the workflow of the bioMérieux EpiSeq ${ }^{\circledR}$ solution and BioNumerics platform in order to confirm or refute the epidemiological link between different MSSA strains.

\section{METHODS}

\section{Case description}

The Antoine Béclère university medical centre includes an academic, tertiary referral unit with 28 neonatal intensive care cots. All infants admitted to the NICU are screened for MSSA and MRSA carriage on anterior nare swabs on admission and then weekly thereafter until discharge. During the first quarter of 2016 (January, February and March) an increase in MSSA bacteraemia was detected in the NICU with an incidence of $3.8 \%(5 / 130)$ as compared with the same period in 2015 , incidence of $0.9 \%(2 / 213)$. This increase triggered a genomic investigation of colonization and bloodstream isolates in order to determine whether we were facing an outbreak. We sequenced the 4 bacteraemia isolates as well as $27 \mathrm{~S}$. aureus colonization isolates from 25 patients obtained between January 2016 and July 2016 (Figure 1). Isolates are labelled with the patient's number identified with a $B$, if the isolate came from a bacteraemia or a $\mathrm{C}$ from a nasal colonization. To explore the possible 
relationship between bacteraemia isolates, we also sequenced the 3 MSSA isolates in blood cultures from 2015 (2015B1, 2015B2, 2015B3).

\section{S. aureus isolation, identification, and drug-susceptibility testing}

S. aureus was detected in blood cultures and nasal swabs used to screen for $S$. aureus colonization on blood agar plates (bioMérieux) after incubation for $24-48 \mathrm{~h}$ at $37^{\circ} \mathrm{C}$ in an ambient atmosphere. Bacterial identification was confirmed by mass spectrometry (MALDI-TOF) (Brucker, Leipzig, Germany). Antimicrobial susceptibility testing was performed using the agar disk diffusion method on Mueller-Hinton (MH, Bio-Rad, Hercules, CA, USA) as recommended [11].

\section{Whole genome sequencing and bioinformatics analysis}

The BioMerieux EpiSeq ${ }^{\circledR}$ solution included the different steps listed in the introduction. This full-service costs $300 €$ per sample, with a guaranteed turnaround time of 7 days starting from isolate reception at the sequencing facility. Isolates should be sent on a Copan swab after a $24 \mathrm{H}$ incubation on blood agar. Then as an element of the EpiSeq workflow, DNA libraries are prepared using the Nextera ${ }^{\circledR}$ XT DNA library preparation kit (Illumina, San Diego, CA, USA). Sequencing is performed on a MiSeq (Illumina) instrument to generate 200 bp paired end reads. Molecular resistance and virulence determinants, MLST and spa typing are inferred from whole genome sequencing (WGS) data through the EpiSeq ${ }^{\circledR}$ knowledge base and data analysis workflow (https://biomerieux-episeq.com). Initial phylogenetic analysis is also conducted directly from WGS data. All these results are made available to the customer through a user-friendly web platform. 
Sequenced genomes of our isolates were also processed for more phylogenetic analysis in this study on the BioNumerics v7.6 (Applied Maths, Sint-Martens-Latem, Belgium). BioNumerics is a unique software platform for integrated analysis of bioinformatics, which has the ability to combine information from various genomic and phenotypic sources into a global database and to conduct conclusive analysis. A de novo assembly was performed on a cloud-based calculation engine with a SPAdes assembler version 3.7.1 [12]. Gene prediction and annotation of the contigs were carry out using BioNumerics. Whole genome MLST (wgMLST) was performed on 3897 loci (core and accessory genes) on the cloud-based calculation engine of BioNumerics v7.6 [12]. To further determine variation among isolates clustered in the same sequence type (ST), we selected as a reference the strain first isolated in 2016 from ST146 and ST30 (isolates 2C and 3B for the ST146 and ST30 lineages respectively). The reads from each isolate in the group were mapped to this reference and focused on single nucleotide polymorphisms in these mapped sequences (wgSNPs). Clustering of the wgMLST and wgSNP results was performed by an unweighted pair group method with arithmetic means-based tree. A minimum spanning tree based on pairwise wgSNP differences was also constructed using BioNumerics.

\section{RESULTS}

\section{Antibiotic resistance profiles of $S$. aureus isolates}

All $S$. aureus isolates responsible for a bacteraemia were meticillin-susceptible and had the same antibiotic susceptibility profile with a resistance only to penicillin G (Figure 2). Colonizing S. aureus isolates were also mainly MSSA. Some strains however had additional resistance mechanisms to macrolides.

\section{Genomic investigation}


Overall, 9 different STs and 13 different spa types were found among the 31 isolates from the study period with three predominant ST (Figure 2). Among these, 11 isolates from 7 patients belonged to the ST146 spa type t002. Five isolates from 4 patients belonged to the ST30 and 4 isolates from 4 patients belonged to the ST398. Assessment of clonal relationships with the wgMLST approach confirmed the clustering among ST146 and ST30 isolates. One additional isolate (20C), with a new ST, clustered with the 4 ST398 isolates in the wgMLST-based dendrogram (Figure 2). The phylogenetic relationship was not further investigated for the ST398 given that the allelic pairwise difference in wgMLST analysis was between 20 and 209. As to isolates from bacteraemia only, they belonged to 3 different STs for 2016 isolates and 2 additional STs for 2015 isolates (Figure 2).

To further explore the relationship between isolates that belong to ST30 we performed a wgSNP analysis (Figure 1S, see online supplementary material). The 3 strains (8B, 2015B2, 3B) isolated from blood cultures had a great number of SNPs with 402 to 435 pairwise differences (Figure 1S). In contrast, strains isolated in blood culture (8B) and a carriage swab (8C) in the same patient, had a difference of only 1 SNP.

The 11 isolates that clustered in ST146 had a maximum pairwise difference of 7 SNPs (Figure 3B, 3C). The ST146 isolate from a blood culture (6B) was identical (0 SNPs) to the colonization strain (6C) isolated on the same day in Patient 6 . Three of the ST146 isolates were recovered from Patient $2(2 \mathrm{C}, 2 \mathrm{C} 1,2 \mathrm{C} 2)$ who was hospitalized in the NICU from December 2015 to August 2016 (Figure 1). S. aureus carriage was detected during almost the entire length of this patient's stay. The sequenced isolates did not differ (0 SNPs) through January and February and only differed 3 SNPs in July. Patient 4 had 2 identical colonization strains (0 SNPs) that were recovered during hospitalization.

\section{Resistome and virulome analysis}


Analysis of antimicrobial resistance determinants (ARD) was concordant with the phenotypic antibiotic resistance profile (Figure 1 and Figure 2S, see online supplementary material). The blaZ gene coding the resistance to penicillin G was the only ARD present in the majority of the isolates. However, different alleles of this gene could be identified and were associated with isolates that belonged to the 3 main groups (ST146, ST30 and ST398). The ermT gene was present in each of the 5 isolates of the ST398 group predicting resistance to erythromycin which was phenotypically associated with an inducible $\mathrm{MLS}_{\mathrm{B}}$ phenotype. Other ARDs were present in the 3 MRSA isolates included in our study with the mecA gene for meticillin resistance, $\mathrm{APH}(6)$ and $\operatorname{aac}\left(6^{\prime}\right)$-aph(2") for aminoglycoside resistance, msrA for erythromycin resistance, fusC for fusidic acid resistance, $d f r G$ for trimethoprim resistance and tetK for tetracycline resistance. Resistance to fluoroquinolone and rifampicin was associated with known point mutations in parC and rpoB respectively (Figure 2S). Of note, all ST30 isolates harboured the mutation Y737F in the rpoB gene, which has been described in vancomycin intermediate S. aureus (VISA) strains [13]. Likewise, isolate $5 \mathrm{C}$ presented a D320N in rpoB, also associated with VISA [14]. Finally, no mupirocin resistance was phenotypically detected nor predicted from WGS data.

Among the virulence determinants, no particular toxin gene and no any identical toxin profile were found in isolates from blood cultures compared to colonization isolates. The tst gene was present in only 2 bacteraemia isolates and the enterotoxin sea gene, which has been associated with sepsis in one study, was found in 4 isolates (Figure 2S). As for antimicrobial resistance, the global profile of toxin genes was associated with the different ST groups (Figure 2S). Only one carriage MRSA isolate (1C) encoded the Panton Valentin leucocidin (PVL). 


\section{DISCUSSION}

Our report focuses on the investigation of an increased number of MSSA bacteraemias in a NICU thanks to the new solution bioMerieux Episeq ${ }^{\circledR}$ and the BioNumerics platform as complementary tools. Indeed, bioMerieux Episeq ${ }^{\circledR}$ is automated and does not require configuration by the user. It is therefore user-friendly. Although BioNumerics allows more indepth analysis and annotation of contigs, it requires settings which entail user expertise and training. The use of the Episeq pipeline enabled us to rule out the suspicion of a global outbreak in the NICU after only 7 days. Indeed, this solution allowed us to obtain MLST, spa type, resistome, virulome and phylogenetic analysis from WGS data in one week for a great number of isolates. This represents an effective time and money saving strategy compared with the time and cost needed to obtain the same results by conventional methods [15].

In our study, we could determine the presence of a polyclonal MSSA population. The presence of various $S$. aureus clones in the NICU over a long period of time was already described for MSSA [16-19]. Using WGS tools, we detected no relationship among the 4 blood culture isolates of 2016, or among those from 2015. These findings demonstrate the absence of patient-to-patient transmission. Consequently, additional contact precautions were taken for patients colonized with MSSA. In addition, a review of hand hygiene, clothing and environmental biocleaning was performed. As a result, a program of educational training (hand hygiene, standard precautions and contact precautions) was set up for the NICU staff by the infection control team. Episeq ${ }^{\circledR}$ results of the polyclonal population of $S$. aureus in the NICU support the initial decision not to resort to staff and environmental screening.

Furthermore, an in-depth analysis with BioNumerics enabled us to identify the transmission of one particular MSSA strain among 7 patients. The clone-specific reference based wgSNP analysis was useful in determining putative transmission events. Indeed, among the 3 major clones, different pairwise wgSNP numbers were observed. Previous studies have determined $S$. aureus mutation rates at 5-10 SNPs a year [20-22]. Moreover, in 
2 studies on $S$. aureus transmission, a cut-off of 30 and 40 SNP differences respectively was used to indicate putative transmission. More precisely, the majority of acquisitions had fewer than 20 SNPs and most had under 4 SNPs $[23,24]$. Accordingly, even if we did not face a single outbreak, we could demonstrate with wgSNP analysis that transmission events occurred for strains of the 3 major clones. Among the ST146 clone, all isolates from different patients had a maximum of 7 pairwise SNP differences. Among ST30, 2 isolates had 23 pairwise SNP differences. wgSNP analysis was not performed for ST398, but wgMLST showed that 2 isolates (19C and 25C) had very few allele differences and could also result from a transmission event. Transmission events of $S$. aureus have previously been linked to patient-to-patient transmission, transmission from healthcare workers or from the environment. Our exclusion of healthcare worker sampling which various articles have pinpointed as a S.aureus reservoir could be considered a weak point in our study $[25,26]$. However Price et al have shown that when standard infection control measures were enforced in an intensive care unit, healthcare workers were not frequent sources of transmission [24]. Our epidemiological study showed that transmission events occurred during 2 distinct periods of time: in February for ST146 and in July for ST146, ST30 and ST398. One patient (Patient 2) carrying the ST146 isolate was present throughout the study period and may have been a reservoir.

WGS contributed to analysis of virulome and resistome, which was concordant with the population structure observed in wgMLST and wgSNP analysis. Isolates responsible for bacteraemia did not have specific toxin genes. Some, but not all, have tst and/or sea genes, which have been correlated with sepsis [27]. Few antimicrobial resistant determinants were detected among the majority of MSSA isolates that correlate with the phenotypic resistance profile and cannot help to discriminate isolates.

wgSNP analysis also showed that blood culture isolates were identical (0 to 1 SNP) to colonization isolates recovered from nasal carriage swabs in the same patient and thus 
confirmed infection was caused by their endogenous strains as already described [28] . Bacteraemia occurred in all patients but one immediately after acquisition of the strain with colonization identified on the same day or a few days before infection.

For now, there is no consensus regarding routine screening for MSSA in neonates. However, given the association of carriage and subsequent infection among this population, screening is likely to be especially beneficial in times of increased incidence, accompanied by the implementation of strategies to prevent the spread of $S$. aureus and to limit new colonization $[5,16,17]$. Recently, Wisgrill et al have demonstrated that active screening and decolonization of neonates cause a decrease in MSSA infections in VLBW children [28]. Decolonization has not been performed in our NICU because the MSSA infection outbreak was brought to a halt after infection control team measures.

Lastly, the BioMerieux EpiSeq ${ }^{\circledR}$ and BioNumerics tools could be very useful in creating a broad epidemiological database to allow the comparison between new isolates and older ones.

\section{CONCLUSION}

For epidemiological purposes, WGS has become the most powerful tool for determining genetic relatedness among strains which help us to effectively combat outbreaks. Moreover, this technology allows both the acquisition of genotyping information and the characterization of the strains (including resistome and virulome). With the availability of benchtop sequencers (such as the new Illumina's iSeq 100), an improved turnaround time for outbreak detection is now feasible now. In conclusion, we have found evidence that the BioMerieux EpiSeq ${ }^{\circledR}$ strategy is time-saving, user friendly and potentially money-saving. 


\section{ACKNOWLEDGMENTS}

This work was supported by bioMerieux S.A. (Marcy l'Etoile, France). We thank Katleen Vranckx, Gaël Kaneko and Bruno H. Muller for assistance in WGS data analysis. We are also grateful to Peggy Haneman-Castex for English editing.

\section{CONFLICT OF INTEREST STATEMENT}

The authors declare no conflict of interest.

\section{FUNDING SOURCE}

The authors have no funding source to declare.

\section{DISCLOSURE}

CR, NBN and FDP have performed the collection, the analysis and the interpretation of data. LR, OR, LP and VD have performed the collection of the data. DDL has performed collection and interpretation of the data. All authors have approved the final article.

\section{REFERENCES}


[1] Greenberg RG, Kandefer S, Do BT, Smith PB, Stoll BJ, Bell EF, et al. Late-onset sepsis in extremely premature infants: 2000-2011. Pediatr Infect Dis J 2017;36:774-9. doi:10.1097/INF.0000000000001570.

[2] Hornik CP, Fort P, Clark RH, Watt K, Benjamin DK, Smith PB, et al. Early and Late Onset sepsis in very-low-birth-weight infants from a large group of neonatal Intensive Care Units. Early Hum Dev 2012;88:S69-74. doi:10.1016/S0378-3782(12)70019-1.

[3] Verstraete E, Boelens J, De Coen K, Claeys G, Vogelaers D, Vanhaesebrouck P, et al. Healthcare-associated bloodstream infections in a neonatal intensive care unit over a 20-year period (1992-2011): trends in incidence, pathogens, and mortality. Infect Control Hosp Epidemiol 2014;35:511-8. doi:10.1086/675836.

[4] Stoll BJ, Hansen NI, Adams-Chapman I, Fanaroff AA, Hintz SR, Vohr B, et al. Neurodevelopmental and growth impairment among extremely low-birth-weight infants with neonatal infection. JAMA 2004;292:2357-65. doi:10.1001/jama.292.19.2357.

[5] Popoola VO, Milstone AM. Decolonization to prevent Staphylococcus aureus transmission and infections in the neonatal intensive care unit. J Perinatol 2014;34:80510. doi:10.1038/jp.2014.128.

[6] Graham PL, Morel A-S, Zhou J, Wu F, Della-Latta P, Rubenstein D, et al. Epidemiology of methicillin-susceptible Staphylococcus aureus in the neonatal intensive care unit. Infect Control Hosp Epidemiol 2002;23:677-82. doi:10.1086/501993.

[7] Shane AL, Hansen NI, Stoll BJ, Bell EF, Sánchez PJ, Shankaran S, et al. Methicillinresistant and susceptible Staphylococcus aureus bacteremia and meningitis in preterm infants. Pediatrics 2012;129:e914-922. doi:10.1542/peds.2011-0966.

[8] Roisin S, Gaudin C, De Mendonça R, Bellon J, Van Vaerenbergh K, De Bruyne K, et al. Pan-genome multilocus sequence typing and outbreak-specific reference-based single nucleotide polymorphism analysis to resolve two concurrent Staphylococcus aureus outbreaks in neonatal services. Clin Microbiol Infect 2016;22:520-6. doi:10.1016/j.cmi.2016.01.024.

[9] Price JR, Cole K, Bexley A, Kostiou V, Eyre DW, Golubchik T, et al. Transmission of Staphylococcus aureus between health-care workers, the environment, and patients in an intensive care unit: a longitudinal cohort study based on whole-genome sequencing. Lancet Infect Dis 2017;17:207-14. doi:10.1016/S1473-3099(16)30413-3.

[10] Lindsey RL, Pouseele H, Chen JC, Strockbine NA, Carleton HA. Implementation of Whole Genome Sequencing (WGS) for identification and characterization of Shiga Toxin-Producing Escherichia coli (STEC) in the United States. Front Microbiol 2016;7. doi:10.3389/fmicb.2016.00766. 
[11] The European Committee on Antimicrobial Susceptibility. Breakpoint tables for interpretation of MICs and zone diameters 2018, 2018.

[12] Earls MR, Kinnevey PM, Brennan GI, Lazaris A, Skally M, O'Connell B, et al. The recent emergence in hospitals of multidrug-resistant community-associated sequence type 1 and spa type 127 methicillin-resistant Staphylococcus aureus investigated by whole-genome sequencing: Implications for screening. PLOS ONE 2017;12:e0175542. doi:10.1371/journal.pone.0175542.

[13] Hiramatsu K, Kayayama Y, Matsuo M, Aiba Y, Saito M, Hishinuma T, et al. Vancomycin-intermediate resistance in Staphylococcus aureus. Journal of Global Antimicrobial Resistance 2014;2:213-24. doi:10.1016/j.jgar.2014.04.006.

[14] Matsuo M, Cui L, Kim J, Hiramatsu K. Comprehensive identification of mutations responsible for heterogeneous vancomycin-intermediate Staphylococcus aureus (hVISA)-to-VISA conversion in laboratory-generated VISA strains derived from hVISA clinical strain Mu3. Antimicrob Agents Chemother 2013;57:5843-53. doi:10.1128/AAC.00425-13.

[15] Nutman A, Marchaim D. "How to do it"-molecular investigation of a hospital outbreak. Clin Microbiol Infect 2018. doi:10.1016/j.cmi.2018.09.017.

[16] Conceição T, Aires de Sousa M, Miragaia M, Paulino E, Barroso R, Brito MJ, et al. Staphylococcus aureus reservoirs and transmission routes in a Portuguese neonatal intensive care unit: a 30-month surveillance study. Microb Drug Resist 2012;18:116-24. doi:10.1089/mdr.2011.0182.

[17] Gomez-Gonzalez C, Alba C, Otero JR, Sanz F, Chaves F. Long Persistence of methicillin-susceptible strains of Staphylococcus aureus causing sepsis in a neonatal intensive care unit. J Clin Microbiol 2007;45:2301-4. doi:10.1128/JCM.00019-07.

[18] Mernelius S, Löfgren S, Lindgren P-E, Matussek A. The role of broth enrichment in Staphylococcus aureus cultivation and transmission from the throat to newborn infants: results from the Swedish hygiene intervention and transmission of $S$. aureus study. Eur J Clin Microbiol Infect Dis 2013;32:1593-8. doi:10.1007/s10096-013-1917-6.

[19] Silva H de A, Pereira EM, Schuenck RP, Pinto RCM, Abdallah VOS, Santos KRN, et al. Molecular surveillance of methicillin-susceptible Staphylococcus aureus at a neonatal intensive care unit in Brazil. Am J Infect Control 2009;37:574-9. doi:10.1016/j.ajic.2008.10.021.

[20] Harris SR, Feil EJ, Holden MTG, Quail MA, Nickerson EK, Chantratita N, et al. Evolution of MRSA During Hospital Transmission and Intercontinental Spread. Science 2010;327:469-74. doi:10.1126/science.1182395. 
[21] Nübel U, Dordel J, Kurt K, Strommenger B, Westh H, Shukla SK, et al. A Timescale for evolution, population expansion, and spatial spread of an emerging clone of methicillinresistant Staphylococcus aureus. PLoS Pathog 2010;6 : e1000855. doi:10.1371/journal.ppat.1000855.

[22] Young BC, Golubchik T, Batty EM, Fung R, Larner-Svensson H, Votintseva AA, et al. Evolutionary dynamics of Staphylococcus aureus during progression from carriage to disease. Proc Natl Acad Sci U S A 2012;109:4550-5. doi:10.1073/pnas.1113219109.

[23] Long SW, Beres SB, Olsen RJ, Musser JM. Absence of patient-to-patient intrahospital transmission of Staphylococcus aureus as determined by whole-genome sequencing. MBio 2014;5:e01692-01614. doi:10.1128/mBio.01692-14.

[24] Price JR, Golubchik T, Cole K, Wilson DJ, Crook DW, Thwaites GE, et al. WholeGenome Sequencing shows that patient-to-patient transmission rarely accounts for acquisition of Staphylococcus aureus in an intensive care unit. Clin Infect Dis 2014;58:609-18. doi:10.1093/cid/cit807.

[25] Layer F, Sanchini A, Strommenger B, Cuny C, Breier A-C, Proquitté H, et al. Molecular typing of toxic shock syndrome toxin-1- and Enterotoxin A-producing methicillinsensitive Staphylococcus aureus isolates from an outbreak in a neonatal intensive care unit. International Journal of Medical Microbiology 2015;305:790-8. doi:10.1016/j.jmm.2015.08.033.

[26] Harris SR, Cartwright EJ, Török ME, Holden MT, Brown NM, Ogilvy-Stuart AL, et al. Whole-genome sequencing for analysis of an outbreak of meticillin-resistant Staphylococcus aureus: a descriptive study. Lancet Infect Dis 2013;13:130-6. doi:10.1016/S1473-3099(12)70268-2.

[27] Xu SX, McCormick JK. Staphylococcal superantigens in colonization and disease. Front Cell Infect Microbiol 2012;2.:52 doi:10.3389/fcimb.2012.00052.

[28] Wisgrill L, Zizka J, Unterasinger L, Rittenschober-Böhm J, Waldhör T, Makristathis A, et al. Active surveillance cultures and targeted decolonization are associated with reduced methicillin-susceptible Staphylococcus aureus infections in VLBW Infants. Neonatology 2017;112:267-73. doi:10.1159/000477295. 


\section{FIGURE LEGENDS}

Figure 1. Timeline of $S$. aureus isolation in carriage swab and blood culture during the 7 months of the study period. Isolates are labelled with the patient's number associated with a $\mathrm{B}$, if the isolate came from a bacteraemia or $\mathrm{C}$ from a nasal colonization

Figure 2. wgMLST-based dendrogram combined with collection date, molecular typing and antimicrobial susceptibility results. Branches corresponding to blood cultures and colonization isolates are shown respectively in red and blue in an unweighted pair group method with arithmetic means-based tree, with results clipped at 200-locus differences. The scale at the top and numbers at nodal positions refer to numbers of wgMLST allelic differences. PEN: penicillin, MET: meticillin, GEN : gentamicin, TOB : tobramycin, TET : tetracyclin, CIP : ciprofloxacin, ERY : erythromycin, CLI : clindamycin, LZD : linezolid, MUP : mupirocin, SXT : trimethoprim-sulfamethoxazole, FA: fusidic acid, RIF: rifampicin

Figure 3. Dissection of ST146 isolates based on wgSNP analysis on an ST146 specific reference $(B$ and $C)$ and isolation timeline of the isolates of this group $(A)$. (B) Circles corresponding to blood cultures and colonization isolates are shown respectively in red and blue in a minimum spanning tree (MST). The numbers on branches indicate wgSNP distances between isolates. (C) Branches corresponding to blood cultures and colonization isolates are shown respectively in red and blue in an unweighted pair group method with arithmetic means-based tree. Numbers in the body of the table represent the number of pairwise wgSNPs between isolates. 


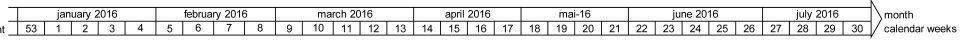
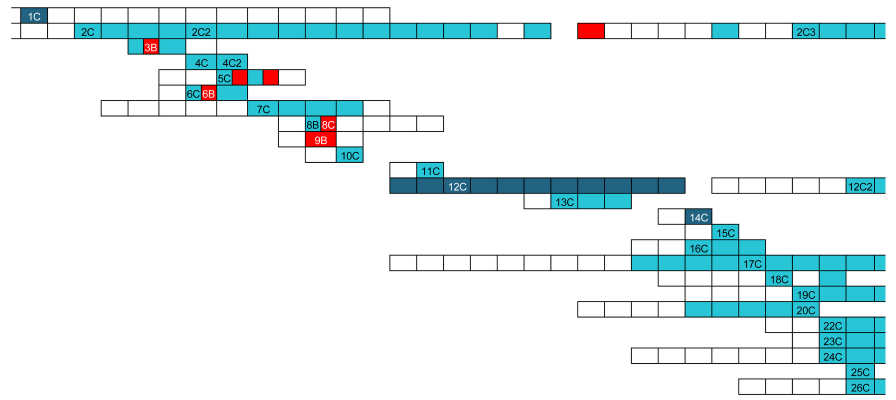

Baby hospitalized in ICU

S. aureus carriage swab positive

$1 C$ S. aureus carriage swab positive sequenced

S. aureus bacteraemia

18 S. aureus bacteraemia sequenced

MRSA carriage

1C MRSA carriage sequenced 
욘 운

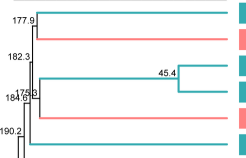

isolate date MLST spa

15C 20/06/16 ST15 t084 2015B1 16/03/15 ST12 t213

13C $09 / 05 / 16$ NA t224

14C 13/06/16 ST97 $\mathrm{t} 359$

2015B3 13/08/15 ST9 t1900

1C $02 / 01 / 16$ NA t121

11C 04/04/16 ST101 t056

18C 04/07/16 ST5 t002

$14 / 03 / 16$ ST5 t002

$11 / 04 / 16$ ST5 NA

14/02/16 ST146 t002

6B $\quad 14 / 02 / 16$ ST146 t002

2C2 08/02/16 ST146 t002

2C $\quad 11 / 01 / 16$ ST146 t002

21C 12/07/16 ST146 t002

26C 25/07/16 ST146 t002

22C $18 / 07 / 16$ ST146 t002

25/07/16 ST146 t002

17C 28/06/16 ST146 t002

4C2 15/02/16 ST146 t002

$4 \mathrm{C}$

12/02/16 ST146 t002

$28.2 \square \quad 3 B$

30/01/16 ST30 t021

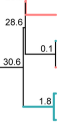

2015B2 03/05/15 ST30 t021

$\begin{array}{llll}8 \mathrm{C} & 07 / 03 / 16 & \mathrm{ST} 30 & \mathrm{t} 1239\end{array}$ 12/03/16 ST30 t1239

$8 \mathrm{~B}$

$24 \mathrm{C}$

$16 \mathrm{C}$

18/07/16 ST30 t021

13/06/16 ST30 t021

$25 \mathrm{C}$

25/07/16 ST398 t12587

200.0

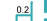
$14.2 \prod^{0.2} 19 \mathrm{C}$ 11/07/16 ST398 t12587 19/07/16 ST398 t13960 R 11/07/16 NA t571 22/02/16 ST398 t571 12/03/16 ST22 t790 $9 \mathrm{~B}$ $5 \mathrm{C}$ 15/02/16 ST45 t331
PEN MET GENTOBTET CIP ERY CLI LZD MUPSXT FA RIF

$\begin{array}{lllllllllllll}R & S & S & S & R & S & S & S & S & S & R & S & S\end{array}$
$\begin{array}{lllllllllllll}R & S & S & S & S & S & S & S & S & S & S & S & S\end{array}$ $\begin{array}{lllllllllllll}S & S & S & S & S & S & S & S & S & S & S & S & S\end{array}$ $\begin{array}{lllllllllllll}R & R & R & R & S & S & S & S & S & S & S & R & S\end{array}$ $\begin{array}{lllllllllllll}R & S & S & S & S & S & S & S & S & S & S & S & S\end{array}$ $\begin{array}{lllllllllllll}R & R & S & R & R & R & R & S & S & S & S & S & S\end{array}$ $\begin{array}{lllllllllllll}S & S & S & S & S & S & S & S & S & S & S & S & S\end{array}$ $\begin{array}{lllllllllllll}R & S & S & S & S & S & S & S & S & S & S & S & S\end{array}$ $\begin{array}{lllllllllllll}R & S & S & S & S & S & S & S & S & S & S & S & S\end{array}$ $\begin{array}{lllllllllllll}R & R & S & S & S & S & S & S & S & S & R & S & R\end{array}$ $\begin{array}{lllllllllllll}R & S & S & S & S & S & S & S & S & S & S & S & S\end{array}$ $\begin{array}{lllllllllllll}R & S & S & S & S & S & S & S & S & S & S & S & S\end{array}$ $\begin{array}{lllllllllllll}R & S & S & S & S & S & S & S & S & S & S & S & S\end{array}$ $\begin{array}{lllllllllllll}R & S & S & S & S & S & S & S & S & S & S & S & S\end{array}$ $\begin{array}{lllllllllllll}R & S & S & S & S & S & S & S & S & S & S & S & S\end{array}$ $\begin{array}{lllllllllllll}R & S & S & S & S & S & S & S & S & S & S & S & S\end{array}$ $\begin{array}{lllllllllllll}R & S & S & S & S & S & S & S & S & S & S & S & S\end{array}$ $\begin{array}{lllllllllllll}R & S & S & S & S & S & S & S & S & S & S & S & S\end{array}$ $\begin{array}{lllllllllllll}R & S & S & S & S & S & S & S & S & S & S & S & S\end{array}$ $\begin{array}{lllllllllllll}R & S & S & S & S & S & S & S & S & S & S & S & S\end{array}$ $\begin{array}{lllllllllllll}R & S & S & S & S & S & S & S & S & S & S & S & S\end{array}$ $\begin{array}{lllllllllllll}R & S & S & S & S & S & S & S & S & S & S & S & S\end{array}$ $\begin{array}{lllllllllllll}R & S & S & S & S & S & S & S & S & S & S & S & S\end{array}$ $\begin{array}{lllllllllllll}R & S & S & S & S & S & S & S & S & S & S & S & S\end{array}$ $\begin{array}{lllllllllllll}R & S & S & S & S & S & S & S & S & S & S & S & S\end{array}$ $\begin{array}{lllllllllllll}R & S & S & S & S & S & S & S & S & S & S & S & S\end{array}$ $\begin{array}{lllllllllllll}R & S & S & S & S & S & S & S & S & S & S & S & S\end{array}$ $\begin{array}{lllllllllllll}S & S & S & S & S & S & R & S & S & S & S & S & S\end{array}$ $\begin{array}{lllllllllllll}S & S & S & S & S & S & R & S & S & S & S & S & S\end{array}$ $\begin{array}{lllllllllllll}R & S & S & S & S & S & R & S & S & S & S & S & S\end{array}$ $\begin{array}{lllllllllllll}S & S & S & S & S & S & R & S & S & S & S & S & S\end{array}$ $\begin{array}{lllllllllllll}R & S & S & S & S & S & R & S & S & S & S & S & S\end{array}$ $\begin{array}{lllllllllllll}R & S & S & S & S & S & S & S & S & S & S & S & S\end{array}$ $\begin{array}{lllllllllllll}R & S & S & S & S & S & S & S & S & S & S & S & S\end{array}$ 


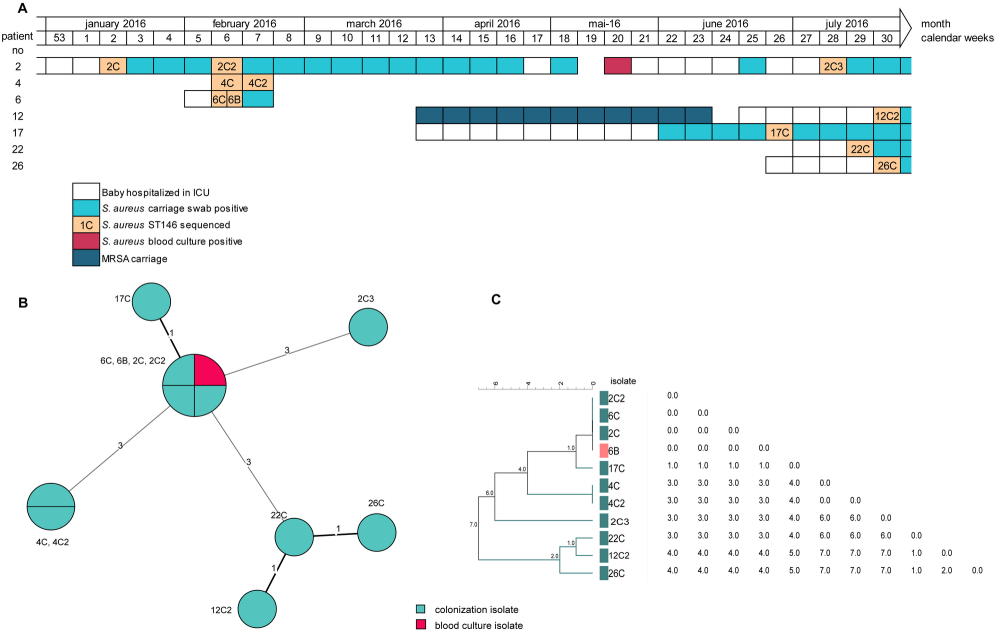




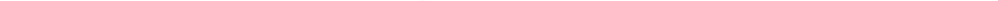


